\title{
Clinical study on the Minimally Invasive Treatment of Early Necrosis of the Femoral Head with Hydroxyapatite Coated Hollow Titanium Rod Supporting the Femoral Head to Prevent Collapse
}

\section{Xinmin Yang ( $\nabla$ yxm11200@126.com )}

The First Affiliated Hospital of Hebei North University https://orcid.org/0000-0002-3209-9100

\section{Ye Tian}

The First Affiliated Hospital of Hebei North University

\section{Yao Yao}

The First Affiliated Hospital of Hebei North Hospital

\section{Research article}

Keywords: Osteonecrosis of the femoral head, Minimally invasive, Protect femoral head treatment, Titanium rob, Clinical efficacy

Posted Date: February 17th, 2021

DOl: https://doi.org/10.21203/rs.3.rs-241604/v1

License: (c) (1) This work is licensed under a Creative Commons Attribution 4.0 International License.

Read Full License 


\section{Abstract}

Background: To investigate the treatment effect of minimally invasive treatment of early osteonecrosis of femoral head (ONFH) with hydroxyapatite coated hollow titanium rod.

Methods: From 1, January 2003 to 1, January 2019, 72 patients with ONFH囚 Stage were selected. There were 50 males and 20 females, aged from 28 to 56 . Onset time was $8 \sim 28$ months. Lesion site: 18 left, 38 right, 16 bilateral. Causes of ONFH: 30 alcohol, 28 hormone, 9 trauma and 5 idiopathic patients. ARCO stage: 14 IIA, 33 IIB, 25 IIC. All patients underwent arthroscopic assisted minimally invasive percutaneous core decompression and bone grafting with hydroxyapatite coated titanium rod surgery. Visua Analogue Scales (VAS), Harris score and Images were used for assessing pain, hip joint function and the stability, respectively. Hip replacement was performed finally.

Results: 16 patients with heavy hormone use history and femoral head collapse underwent Total Hip

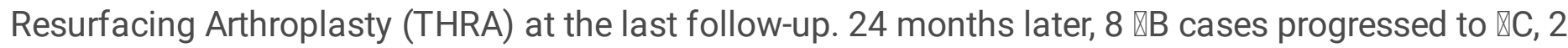

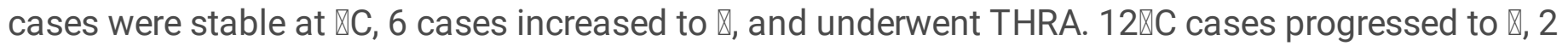

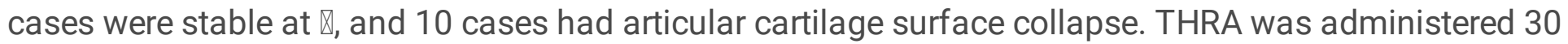
months after surgery. VAS score of individual patients increased and Harris score decreased 24 months after surgery, but there was no significant difference between the scores of 12 months and 24 months. The clinical effect of the last follow-up showed that the postoperative improvement rate of this group was $76.13 \%$, among which the best was $100 \%$ in IIA, $79.48 \%$ in IIB, and the lowest was 58.06 in IIC. Both the patients with IIB or IIC, cases with aggravation and without change were hormonal ONFH.

Conclusion: The treatment of ONFH with hydroxyapatite coated hollow titanium rod supporting the femoral head to prevent collapse is feasible. In addition to the Glucocorticoid-induced ONFH stage $\mathbb{} \mathrm{C}$

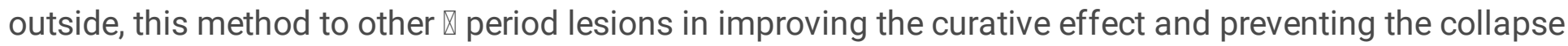
of femoral head articular surface is good and safe.

\section{Introduction}

In recent years, the incidence of osteonecrosis of the femoral head (ONFH) is increasing and the patients is younger. When ONFH is not effectively treated in the early stage, the biomechanical properties of the subchondral bone in the weight-bearing region of the femoral head gradually changed with the process of osteonecrosis. When the stress increased to a certain level, the subchondral bone trabecular fracture occurs in the collapse of the femoral head and the hip joint function was severe limited[1].There are many treatments to handle early ONFH, but the common goal is to preserve the femoral head and avoid or delay hip replacement. In many head preserving treatment methods, effectively avoiding or preventing femoral head collapse is the goal of ONFH treatment in the early stage [2-5]. Therefore, from January 2003 to 1 December 2018, the author used hydroxyapatite coated titanium rod designed by himself to support the femoral head and prevent collapse to treat ONFH. The rod could effectively provide mechanical support, improve the biomechanical properties of the femoral head and neck, and prevent the 
collapse of the femoral head. The clinical results and follow-up confirmed the effect of this rod. Thus, the data were showed as follows.

\section{Materials And Methods}

\subsection{Selectioncriteria}

Case inclusion criteria: According to the Association Research Circulation Osseous (ARCO) staging standard, adult patients with stage II ONFH due to heavy hormone use, long-term alcohol abuse, trauma and idiopathic factors. Case exclusion criteria: (1) Immature bone development; (2) ONFH at phase I, III and IV; (3) Application of immunosuppressive agents; (4) Surgical history of femoral neck fracture; (5) History of hip infection; (6) Complete clinical follow-up data are lacking.

\subsection{Research objects}

The clinical data of 72 patients with stage II ONFH who met the selection criteria from 1 January 2003 to 1 December 2018 were retrospectively analyzed. There were 52 males and 20 females, aged from 28 to 56 years, with an average age of $37.9 \pm 4.8$. 18 cases were at the left side, 38 cases were at the right side, 16 cases were at both sides, and a total of 88 femoral heads were included. The time course of the disease ranged from 8 to 28 months, with an average of $16.7 \pm 1.5$ months. 72 patients underwent comprehensive physical examination after admission to identify surgical risk factors, and preoperative targeted treatments of internal medical diseases was applied normally to control and stabilize the internal medical condition. Arthroscopically assisted minimally invasive percutaneous core decompression, bone grafting and hydroxyapatite coated titanium rod were performed to support the femoral head. This clinical treatment program was authorized and approved by the ethics committee of the first affiliated hospital of Hebei Northern University (Batch No.: 2003-01). All patients and their families were informed of the implementation of the treatment program and signed the informed consent. The corresponding table of ONFH pathogenic causes and ARCO stages were shown in Table 1.

Table 1 Causes of ONFH in 72 patients ( 88 femoral heads) and ARCO stage before treatment (Numberofcases(The femoral head)) 


\begin{tabular}{|lll|}
\hline Causes & $\begin{array}{l}\text { Number of cases } \\
\text { (The femoral head) }\end{array}$ & ARCO stage \\
& Alcohol Astage IIB stage II Cstage \\
\cline { 1 - 1 } & $30(35)$ & $4(5) 14(18) 12(12)$ \\
\hline hormone & $28(35)$ & $7(8) 11(15) 10(12)$ \\
\hline$\underline{\text { trauma }}$ & $9(10)$ & $2(3) 3(3) 4(4)$ \\
\hline$\underline{\text { idiopathic }}$ & $5(8)$ & $1(2) 2(3) 2(3)$ \\
\hline subtotal & $72(88)$ & $14(18) 30(39) 28(31)$ \\
\hline
\end{tabular}

\subsection{Hydroxyapatite coated titanium rod}

China National Invention Patent ZL 201120071962.8 and utility model patent ZL 200920266161x. The titanium rod is titanium metal coated with hydroxyapatite. The rod diameter is $10 \mathrm{~mm}$, the hollow diameter is $2 \mathrm{~mm}$, and the rod tail length is $10 \sim 20 \mathrm{~mm}$ thick thread. Gradually increase the rod tail length every $5 \mathrm{~mm}$. Thread core diameter $10 \mathrm{~mm}$, thread outside diameter $11 \mathrm{~mm}$, thread spacing $1.5 \mathrm{~mm}$. The length of the rod is $60 \sim 115 \mathrm{~mm}$, and the length of the rod is gradually increased every $2 \mathrm{~mm}$. The length of the rod tail corresponding to the whole length of the rod is $60 \sim 70 \times 10 \mathrm{~mm}, 72 \sim 80 \times 15 \mathrm{~mm}, 82 \sim 115 \times 2$ $\mathrm{mm}$. The tail end has an equilateral triangle notch with side length of $6 \mathrm{~mm}$ (FIG.1-2).

\subsection{Surgical methods}

Intravertebral anesthesia, the patient was placed in supine position with high hip pad. C-arm X-ray machine was used for perspective positioning. The $2 \mathrm{~mm}$ guide needle was drilled parallel to the axis of the femoral neck at $3 \sim 4 \mathrm{~cm}$ below the lateral greater trochanter to the central target in the necrosis area of the femoral head, that is, the subchondral bone of the femoral head was $0.5 \mathrm{~cm}$ (the position was correct by positive and lateral X-ray) (FIG.3 a). The skin was cut longitudinally for $1.5 \mathrm{~cm}$, the muscle layer was separated, and a work tube with a diameter of $1.0 \mathrm{~cm}$ was inserted. $6,8,10 \mathrm{~mm}$ hollow drill was successively drilled through the guide needle to the subchondral area of core decompression (FIG.3 b). The surgery was performed alternately under the guidance of C-arm X-ray fluoroscopy and under the direct observation of arthroscopy through bone tunnel. Special long-handle curette or grinding drill with different angles and different specifications were inserted through the core decompression bone canal to scrape the necrotic bone tissue and sclerotic bone tissue in the cystic lesion area of the femoral head (FIG. 3c). At the same time, arthroscopy was used for observation until there was no collapse of subarticular cartilage bone, the articular surface was intact, the lesion was cleared and normal bone and fresh blood appeared (FIG.4). The bone tunnel and lesion area were rinsed with normal saline under pressure, and the autogenous bone (ilium) was inserted into the curettage area of the femoral head lesion and compressed through the core decompression channel (FIG. 5a-b). Hollow titanium rod coated with hydroxyapatite in appropriate length was selected and inserted into the subchondral of the femoral head (FIG. 5c), and the incision was sutured (FIG.6). All operations were performed by the same surgeon. 


\subsection{Postoperativerehabilitation}

After the operation, all the patients underwent continuous passive exercise (CPM) exercise on the affected lower limb, and the support and protection of double crutches were used not less than 6 months. After unilateral ONFH surgery, the affected limb gradually walked with heavy load after holding both crutches for 3 months. After 3 months in bed after bilateral ONFH, light weight and walk slowly under the protection of two crutches. After 6 months, walk with alternating load on the left and right sides under the protection of the crutches.

\subsection{Postoperative efficacy evaluation index}

Visua Analogue Scales (VAS) were used as indicators for pain assessment. Harris score was used as an indicator of hip joint function. The imaging findings were used as the index to evaluate the stability of the lesion stage. Among them, X-ray evaluated the lesion stage: no progression as stable, progresses within the same staging as instability, progresses to stage III or the femoral head collapses as deterioration. Improvement, no change and aggravation were used as the evaluation indexes of clinical efficacy. Among them, Improvement: VAS score of hip pain and Harris score of hip function were relieved or improved $\geq 50 \%$ compared with preoperative, and X-ray performance was stable. No change: VAS score for hip pain and Harris score for hip function were relieved or improved $<50 \%$ compared with preoperative, and X-ray was stable or unstable. Aggravation: VAS score for hip pain and Harris score of hip joint function were poor score than before surgery, and X-ray performance deteriorated. Hip replacement (Total Hip Resurfacing Arthroplasty (THRA)) was performed as the end point of observation.

\subsection{Statisticaltreatment}

SPSS 15.0 statistical software was used for analysis, measurement data were expressed as $a+b$, and ANOVA was used for inter-group comparison. $T$ test was used for comparison between different time after treatment and before treatment, $a=0.05$ was the test level. The final follow-up or THRA was used as the end point of observation.

\section{Result}

\subsection{The Follow-up situation}

In this study, follow-up time was 24 to 36 months, with an average of (28.4 1.2) months. In the last follow-up, 16 patients underwent THRA in the collapse of the femoral head, all of which had a history of heavy hormone use.

\subsection{X-ray observation of the stability of lesion staging before and after surgery}

Postoperative X-ray observation of ONFH in installment, the postoperative 24 months $\mathbb{B}$ period of 8 cases (8) femoral head lesion is aggravating development to $₫ \mathrm{C}$ period, including 2 cases of lesions stable in $\triangle C$ period, remaining 6 cases continue to increase $\otimes$ periods and worsening, implement THRA. 
24 months postoperatively IIC period 12 cases ( 12 femoral head囚lesion is aggravating, including 2 cases ( 2 femoral head冈lesion in $\nabla$ period stable , 10 cases ( 10 femoral head) deterioration of articular cartilage surface subsidence, implement THRA 30 months after surgery (Table 2).

\section{Table 2. X-ray staging changes before and after surgery}

\begin{tabular}{|llllll|}
\hline \multirow{2}{*}{ Preoperative staging } & Number of cases & \multicolumn{4}{l|}{ Postoperative stage } \\
\cline { 5 - 7 } & (The femoral head) & IIA & IIB & IIC & Q stage \\
IIA stage & $14(18)$ & $14(18)$ & 0 & 0 & 0 \\
\hline IIB stage & $30(39)$ & 0 & $22(31)$ & $2(2)$ & $6(6)$ \\
IIC stage & $28(31)$ & 0 & 0 & $16(19)$ & $12(12)$ \\
\hline
\end{tabular}

\subsection{VAS and Harris scores before and after surgery}

The VAS and Harris scores improved significantly after the operation, and there was a difference between the time points after the operation and before the operation $\mathrm{P}<0.05$, but there was no difference between 24 months after the operation and 12 months after the operation $\mathrm{P}>0.05$ (Table 3 ).

\section{Table 3. VAS and Harris scores before and after surgery}

\begin{tabular}{|c|c|c|c|}
\hline Time & $\begin{array}{l}\text { Number of cases } \\
\text { (The femoral head) }\end{array}$ & VAS score & Harris score \\
\hline preoperative & $72(88)$ & $5.6 \pm 1.1(3 \rrbracket 9)$ & $76.4 \pm 3.6(72 \bowtie 89)$ \\
\hline 6 months after surgery & $72(88)$ & $4.1 \pm 0.9(1 \rrbracket 6)$ & $85.4 \pm 1.12(84 \llbracket 92)$ \\
\hline 12 months after surgery & $72(88)$ & $2.1 \pm 0.3(0 \otimes 6) *$ & $93.8 \pm 2.8(85 \rrbracket 100)$ * \\
\hline 24 months after surgery & $72(88)$ & $2.4 \pm 0.3(0 \otimes 10) *$ & $91.9 \pm 2.4(81 \otimes 100)$ * \\
\hline The last follow-up & $48(64)$ & $0.7 \pm 0.2(0 \rrbracket 2)$ & $98.6 \pm 1.2(95 \rrbracket 100)$ \\
\hline $\mathrm{F}$ & & 141.27 & 354.16 \\
\hline $\mathrm{P}$ & & $\varangle 0.05$ & $\otimes 0.05$ \\
\hline
\end{tabular}

Note: Compared with the preoperative, there was a difference $(P<0.05) .{ }^{*}$ no difference between the two $(P$ $>0.05)$

\subsection{Clinical efficacy evaluation results of the final follow-up}

At the last follow-up, 72 patients ( 88 femoral head) were treated, 51 (67 femoral head) were improved, 2 ( 2 femoral head) were not changed, and 19 (19 femoral head) were aggravated. Finally, 16 patients (16 femoral head) were treated with THRA, all of whom were hormonally ONFH patients. The total 
postoperative improvement rate was $76.13 \%$, and the improvement rates of stage IIA, stagellB and stage IIC were $100 \%, 79.48 \%, 58.06 \%$, respectively. The cases with no change and aggravation were both hormonal ONFH (Table 4).

\section{Table 4 Clinical efficacy of the final follow-up}

\begin{tabular}{|lllllll|}
\hline $\begin{array}{l}\text { Clinical } \\
\text { stages }\end{array}$ & $\begin{array}{l}\text { Femoral head } \\
\text { count }\end{array}$ & $\underline{\text { Improve }}$ & $\begin{array}{l}\text { No } \\
\text { change }\end{array}$ & Aggravate & THRA & $\begin{array}{l}\text { Improvement } \\
\text { rate叉\%区 }\end{array}$ \\
\hline IIA & 18 & 18 & 0 & 0 & 0 & 100 \\
\hline IIB & 39 & 31 & 2 & 6 & 6 & 79.48 \\
\hline IIC & 31 & 18 & 0 & 13 & 10 & 58.06 \\
\hline Total & 88 & 67 & 2 & 19 & 16 & 76.13 \\
\hline
\end{tabular}

\section{Discussion}

\subsection{Domestic and foreign conditions of head preserving treatment for femoral head necrosis}

$\mathrm{ONFH}$ is a multifactorial disease with multiple pathological mechanisms and ultimately the same outcome, resulting in bone marrow cell ischemia and bone cell necrosis. It is a common and difficult disease in orthopedics [6, 7]. For patients with early ONFH, femoral head preservation is the preferred target. The main methods include core decompression combined with or without bone graft, Vascularized Fibula Graft and allograft fibula transplantation or tantalum rod implantation. The clinical results of core decompression surgery are uncertain, lack of structural support, and lack of long-term mechanical support even in combination with bone grafting, resulting in bone collapse [8-10]. Vascularized fibula transplantation requires free peroneal artery and anastomosis with the external femoral artery of the affected hip, which has the disadvantages of the need for enlarged surgical procedure, high morbidity rate in the donor area, long recovery time and the risk of fractures in the proximal femur $[1,11]$. However, allograft fibula transplantation is expensive and may be absorbed and rejected, which will increase the technical difficulty of THRA in the future. The above methods have some defects, such as large trauma and insufficient donor source. However, although the support effect of tantalum rods used recently at home and abroad is clear, the price is high and it is difficult for patients to accept. At present, there is no clear report that tantalum rods can help promote bone repair. Meanwhile, with the in-depth study of tantalum rods, the number of cases of their failure increases gradually and it is difficult to remove the titanium rod $[12,13]$. Therefore, finding a minimally invasive method with the ability to repair the bone defect in the necrotic area of the femoral head and the ability to provide immediate and permanent structural support to prevent the collapse of the femoral head is the key to the early ONFH head preserving treatment [14].

\subsection{Advantages of hydroxyapatite coated hollow titanium rod designed to support the femoral head and prevent collapse}


The purpose and function of hydroxyapatite coated titanium rod is to provide strong and effective mechanical support in the early ONFH bone destruction area and prevent cartilage surface collapse of the femoral head, and to conduct bone grafting at the core decompression area, which is conducive to the biomechanical recovery and bone fusion of the head and neck, without hindering the long-term hip replacement. Hydroxyapatite coated hollow titanium rod has the following design advantages: 『Hollow titanium rod adopts titanium alloy with elastic modulus close to human skeleton, which has good histocompatibility. Compared with tantalum rod, it has low cost and low price, which can reduce the hospitalization cost of patients. $\otimes$ The hollow design of the titanium rod facilitates the entry and exit of the guide needle. During the placement, the guide needle can be used for accurate positioning to achieve precise titanium rod placement, which reduces the damage to bone tissue during positioning. The hollow design forms a channel in a short time and plays a role in decompression. The smooth inner surface of the hollow is not conducive to bone formation and can achieve long-term decompression effectiveness. $\nabla T h e$ smooth rod design of the rod body and the $1 / 3$ circular design of the rod head make the resistance of the rod less. The $1 / 3$ circular design of the rod head corresponds to the circular structure of the femoral head, which can be more closely attached to the subchondral bone of the femoral head. $₫$ The titanium rod can be embedded into the lateral cortical bone by using the thread design of the rod tail. The rod body diameter of titanium rod is $10 \mathrm{~mm}$ and the diameter outside the thick thread is $11 \mathrm{~mm}$. This design with small head and large tail combined with the biting force of the thick thread effectively prevents the rod tail from protruding or the rod head penetrating into the cartilage surface. खThe overall length of titanium rod is $75 \sim 110 \mathrm{~mm}$, increasing every $5 \mathrm{~mm}$, which is in line with the average length of femoral neck in human body, and available for patients of different ages. The end of the rod tail is designed as an internal six-square notch with a depth of $3.5 \mathrm{~mm}$. The Embedded bite contact between the tool and the rod tail with a depth of $3.5 \mathrm{~mm}$ is conducive to the entry or removal of the hollow titanium rod, making the surgical operation easier, less labor and not easy sliding buckle, and according to the custom of patients, most internal fixators are required to be taken out after bone healing, which is also in line with humanized application.

\subsection{Necessity and effect of hollow titanium rod coated with hydroxyapatite on supporting and preventing collapse of femoral head}

The ARCO stage belongs to the early ONFH, and its radiological and histopathological changes have obviously shown osteonecrosis and cystic changes of the femoral head. If the lesion continues to develop and cannot be effectively controlled, it is bound to cause fracture or absorption of the trabecular bone in the weight-bearing area, and the destruction of bone structure in the subchondral area, that is, the reduction of mechanical strength and supporting force in the local weight-bearing area of the femoral head, which will eventually lead to the collapse of the femoral head and ONFH. Clinical studies have found that after ONFH core decompression and lesion clearance, both the remaining femoral neck decompression tunnel and bone defect in the area of femoral head necrosis can cause the original weak femoral head to lack of bone structure support, resulting in stress concentration and accelerated collapse of the cartilage surface of the femoral head. 
With the reconstruction of blood supply, in the process of ONFH repair broken bone and bone resorption is often greater than the speed of new bone formation, although after the lesion clearance on the cavity of the femoral head bone graft, but the implanted bone lacks immediate and permanent mechanical support, continued for a long time in the human body weight under the action of the bone graft only Microsoft support will gradually disappear, further cause and aggravate the collapse of the femoral head [15]. Therefore, after ONFH lesion clearance, bone graft not only fills in the bone defect to restore anatomical tissue structure, accelerates its vascularization to promote bone repair, but also increase the intracavitary mechanical strength of femoral head lesion removal.

Hydroxyapatite coated hollow titanium rod was inserted into the femoral neck of the femoral head and the weight-bearing necrosis area of the femoral head. Hydroxyapatite coating could form a firm bone bond at the interface between the titanium rod and bone, and the irregular fracture zone in the femoral head was stabilized. To improve the biomechanical characteristics of the femoral head neck, provide structural and mechanical permanent support for the subchondral site where the articular surface of the femoral head may collapse. The latter support is conducive to recovery and plays an important role in maintaining the concentric circle structure corresponding to the acetabulum and the femoral head [1] (See figure 2) (FIG.7)

\subsection{Feasibility analysis and efficacy evaluation of hollow titanium rod coated with hydroxyapatite for ONFH head preserving treatment}

The changes in ONFH staging were observed by X-ray at 24 months after surgery. Table 1, table 2 and follow-up data showed that whether 6 patients with stage IIB or 10 patient with stage IIC were aggravated to stage and deteriorated, Finally, THRA was performed surgically and Its pathogenic factors were hormone. Postoperative $\mathrm{X}$ - ray observation of the other stages of the case stage stability, lesion repair, good support of titanium rod, femoral head articular surface without collapse, no hip degeneration. This indicates that this treatment method has a good effect on patients with stage II ONFH caused by causes other than hormonal ONFH (See table 2) (FIG.8).

According to postoperative pain and hip joint function can be known, both VAS and Harris scores improved significantly at each time point after surgery compared with before surgery, indicating that this treatment can alleviate pain, improve hip joint function and optimize score index. In particular, the early postoperative period (6 12 months) and the late postoperative period (24 months the last follow-up) showed significant improvement.

VAS scores of individual patients increased and Harris scores decreased at 24 months after surgery, but there was no statistically significant difference between them and those at 12 months. Combined with interval personalized data analysis, it was found that there were cases of pain aggravation and hip function was affected during this period (See table 3).

The clinical effect of the last follow-up showed that the postoperative improvement rate of this group was $76.13 \%$, among which the improvement rate of stage IIA was the best $100 \%$, the improvement rate of 
stage IIB was $79.48 \%$, and the improvement rate of stage IIC was the lowest $58.06 \%$. Whether the patients with stage IIB or IIC aggravation and no change were patients with glucocorticoid-induced ONFH. The results showed that this method had the best effect in improving the curative effect and preventing the collapse of femoral head for ONFH stage II A and IIB lesions. For IIC period curative effect sure, but two years later risk of head collapse in glucocorticoid-induced ONFH exists. It is related to the hormone leading to a significant decrease of VEGF and BMP in the trabecular bone and bone marrow tissue of the femoral head and inhibiting the synthesis of BMP by osteoblasts and the differentiation of BMSC into osteoblasts decreased, and adipocyte increased $[16,17]$. Postoperative histopathological observation also confirmed that hormonal ONFH was mainly characterized by bone destruction and absorption and granulation tissue formation, with weak bone repair and osteogenesis ability, and the inhibitory effect of postoperative hormone on osteogenesis activity persisted. That is, progressive bone loss and osteonecrosis occurred, resulting in the loosening of the internal fixation [18-20], and the failure of the titanium rod support caused the collapse of the femoral head. Therefore, the therapeutic effect of ONFH in stage IIC of hormone is relatively poor, so it should be carefully selected, while better clinical effect can be obtained for ONFH lesions caused by trauma, alcohol and idiopathic factors (See table 4).

\section{Conclusions}

The application of hydroxyapatite coated hollow titanium rod to support the femoral head to prevent collapse is the trend of minimally invasive surgical treatment of ONFH. Has the following advantages: small incision, less pain,very few bleeding, accurate positioning and minimally invasive surgery, effective accurate pulp core decompression, lesions cleared completely, reconstruction of bone structure, permanent mechanical support,small interference to biomechanical strength of hip joint, significant pain relief, more accurate and reasonable, easy operation and postoperative function good, fast recovery, obviouscurative effect, low cost, easy to accept patients with, even treatment failure does not affect late

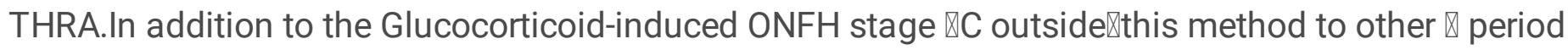
lesions in improving the curative effect and preventing the collapse of femoral head articular surface is good and safe. The number of cases in this study is relatively small, the observation time is relatively short, and there is a selection bias, no control study, still need to be a large sample study and long-term follow-up observation.

\section{Abbreviations}

Osteonecrosis of femoral head ONFH

Association Research Circulation Osseous ARCO

Continuous passive exercise CPM

Visua Analogue Scales VAS

Total Hip Resurfacing Arthroplasty THRA 


\section{Declarations}

\section{Disclosure}

Not applicable

\section{Consent for publication}

Not applicable.

\section{Availability of data and materials}

The datasets used and analysed during the current study are available from the corresponding author on reasonable request.

\section{Competing interests}

The authors declare that they have no competing interests.

\section{Funding}

The study was supported by Mandatory Planning Project of Scientific and Technological Bureau of Zhangjiakou City (2017 (1712005D)) and Science and Technology Project of Guangdong Province (201707010089).

\section{Authors' contributions}

Xinming Yang designed this study, Liangbin Gao wrote the manuscript, Bo Li, Xumin Hu, Di Zhang, Qiancheng Zhao, Xu Jiang and Chi Zhang collected the data, Jionglin Wu and Rui Guo analyzed and interpreted the patient data. Ye Tian and Yao Yao edited the images. All authors read and approved the final manuscript.

\section{Acknowledgements}

Not applicable

\section{References}

1. Xinming Y, Wei S, Yakun D. Analysis of clinical effect of non-cellular tissue engineering bone combined with Titanium rod after minimally invasive grafting in the treatment of early stage osteonecrosis of the femoral head. Acta Mech Sin. 2010;26(1):13-9.

2. Persiani P, De Cristo C, Graci J, et al. Stage-related results in treatment of hip osteonecrosis with coredecompression and autologous mesenchymal stem cells. Acta orthopaedica Belgica. 
2015;81(3):406-12.

3. Woodhouse AG, Drake ML, Lee GC, et al. Free vascularized fibular grafts for femoral head osteonecrosis: alternative technique utilizing a buttress plate for graft fixation. J Surg Orthop Adv. 2015;24(2):144-6.

4. Liu ZH, Guo WS, Li ZR, et al. Porous tantalum rods for treating osteonecrosis of the femoral head. Genet Mol Res. 2014;13(4):8342-52.

5. Tang SB. [Strengthen basic research to improve diagnosis and therapeutics of vitreoretinal disease]. [Zhonghua yan ke za zhi]. Chinese journal of ophthalmology. 2003;39(11):641-4.

6. Aldridge JM 3rd, Urbaniak JR. Avascular necrosis of the femoral head: etiology, pathophysiology, classification, and current treatment guidelines. Am J Orthop (Belle Mead NJ). 2004;33(7):327-32.

7. Lieberman JR, Berry DJ, Mont MA, et al. Osteonecrosis of the hip: management in the 21st century. Instr Course Lect. 2003;52:337-55.

8. Beckmann J, Schmidt T, Schaumburger J, et al. Infusion, core decompression, or infusion following core decompression in the treatment of bone edema syndrome and early avascular osteonecrosis of the femoral head. Rheumatol Int. 2013;33(6):1561-5.

9. Zhao DW, Yu XB. Core decompression treatment of early-stage osteonecrosis of femoral head resulted from venous stasis or artery blood supply insufficiency. J Surg Res. 2015;194(2):614-21.

10. Daltro GC, Fortuna V, de Souza ES, et al. Efficacy of autologous stem cell-based therapy for osteonecrosis of the femoral head in sickle cell disease: a five-year follow-up study. Stem Cell Res Ther. 2015;6(1):110.

11. Fontecha CG, Roca I, Barber I, et al. Femoral head bone viability after free vascularized fibular grafting for osteonecrosis: SPECT/CT study. Microsurgery. 2016;36(7):573-7.

12. Miao H, Ye D, Liang W, et al. Effect of Osteonecrosis Intervention Rod Versus Core Decompression Using Multiple Small Drill Holes on Early Stages of Necrosis of the Femoral Head: A Prospective Study on a Series of 60 Patients with a Minimum 1-Year-Follow-Up. Open Orthop J. 2015;9:179-84.

13. Zhang $X$, Wang J, Xiao J, et al. Early failures of porous tantalum osteonecrosis implants: a case series with retrieval analysis. Int Orthop. 2016;40(9):1827-34.

14. Ye YH, Chen K, Jin KK, et al. [Progress on surgical treatment for femoral head-preservering in the precollapse stage of femoral head necrosis]. Zhongguo Gu Shang. 2017;30(3):287-92.

15. Schneider W, Breitenseher M, Engel A, et al. [The value of core decompression in treatment of femur head necrosis]. Der Orthopade. 2000;29(5):420-9.

16. Zhenqiu C, Wei H, Qiushi W, et al. Research on expression levels of bone metabolism related protein in osseous tissue of glucocorticoidinduced necrosis of femoral head. Chinese Journal of Joint Surgery(Electronic Edition). 2015.

17. Li X, Jin L, Cui Q, et al. Steroid effects on osteogenesis through mesenchymal cell gene expression. Osteoporosis international: a journal established as result of cooperation between the European 
Foundation for Osteoporosis and the National Osteoporosis Foundation of the USA. 2005;16(1):101-8.

18. Simon JP, Berger P, Bellemans J. Total hip arthroplasty in patients less than 40 years old with avascular necrosis of the femoral head. A 5 to 19-year follow-up study. Acta orthopaedica Belgica. 2011;77(1):53-60.

19. Goffin E, Baertz G, Rombouts JJ. Long-term survivorship analysis of cemented total hip replacement (THR) after avascular necrosis of the femoral head in renal transplant recipients. Nephrology, dialysis, transplantation: official publication of the European Dialysis and Transplant Association -. European Renal Association. 2006;21(3):784-8.

20. Rockwood JH, Whiddon DR, Sekiya JK. Arthroscopic Management of Avascular Necrosis. Operative Techniques in Orthopaedics. 2005;15(3):273-9.

\section{Figures}

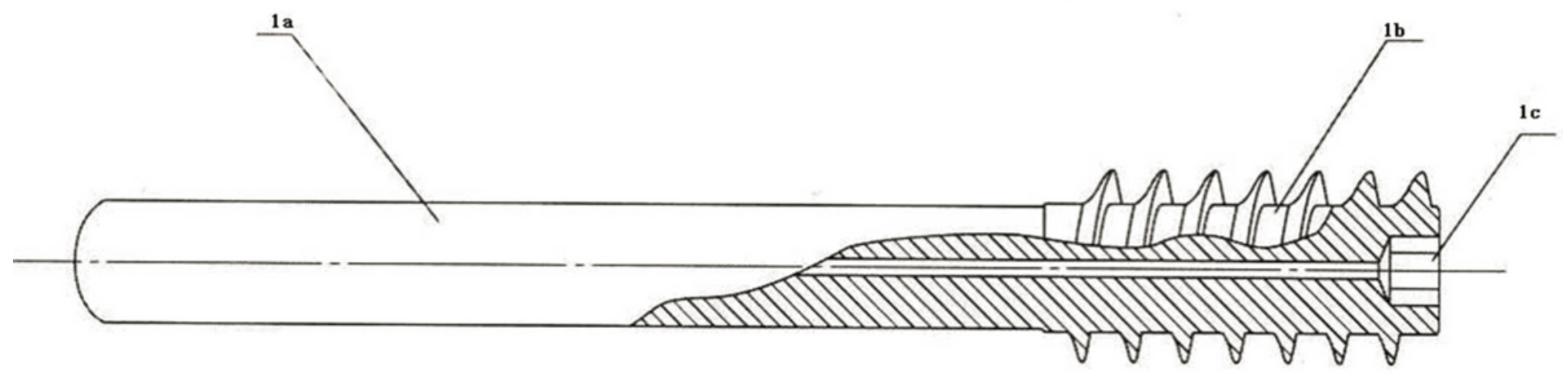

\section{Figure 1}

a. Rod body with hollow through hole; b. Rod tail coarse screw thread; c. Rod tail Hexagon notch
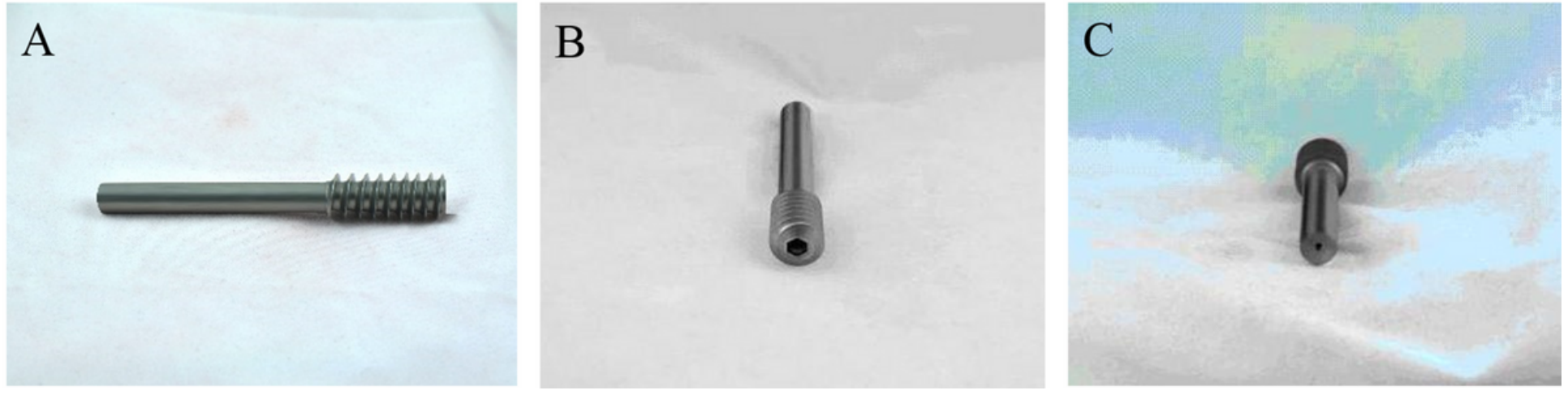

Figure 2

Solid image of titanium rod; a. Rod overall image; b. Rod tail image; c. Rod head image 

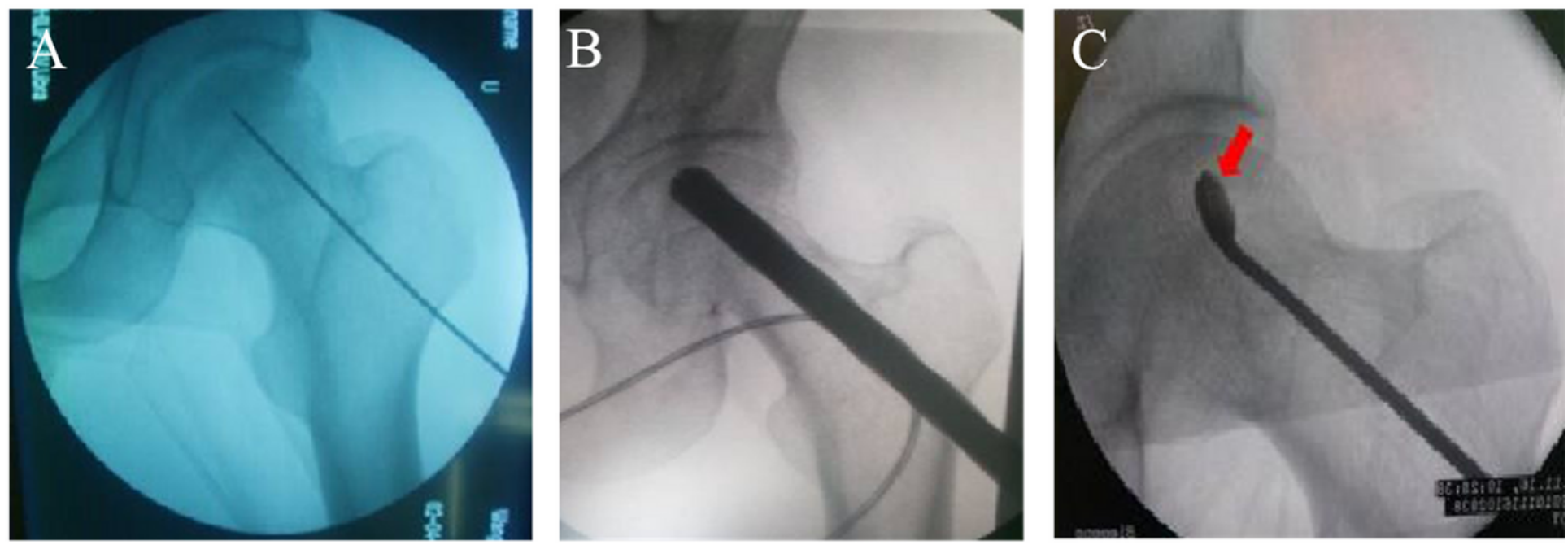

Figure 3

Surgical operation. a. Accurate positioning of the guide pin; b. Core decompression; $c$. Curettage of the lesion.
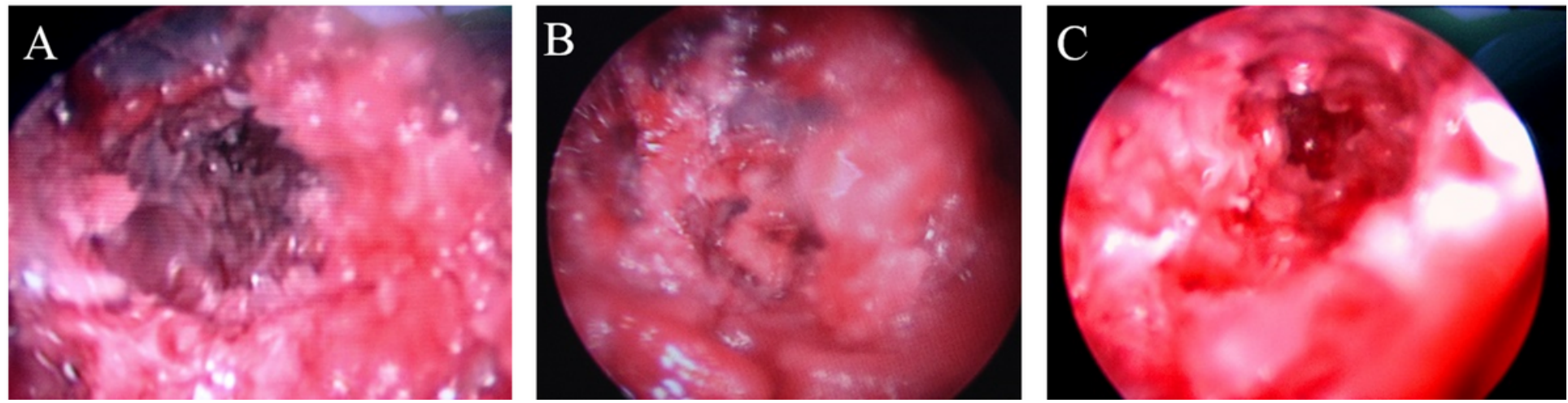

Figure 4

Arthroscopic observation. a. Bone tissue with ischemic necrosis in cystic degeneration of the femoral head; B. Sclerosis bone of ischemic loosening inside the femoral head; C. Blood supply was improved after complete curettage of femoral head lesions.
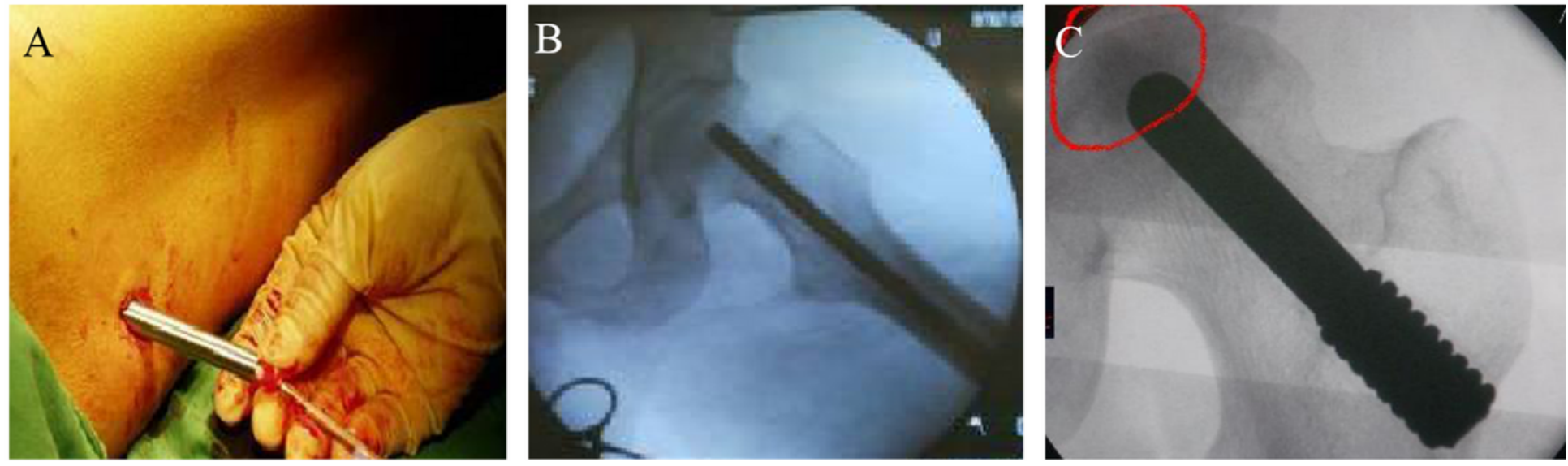

Figure 5 
Surgical operation. a. Bone graft; b. Top bar compaction of bone graft grains; c. Titanium rod placement (The marks are bone implants).

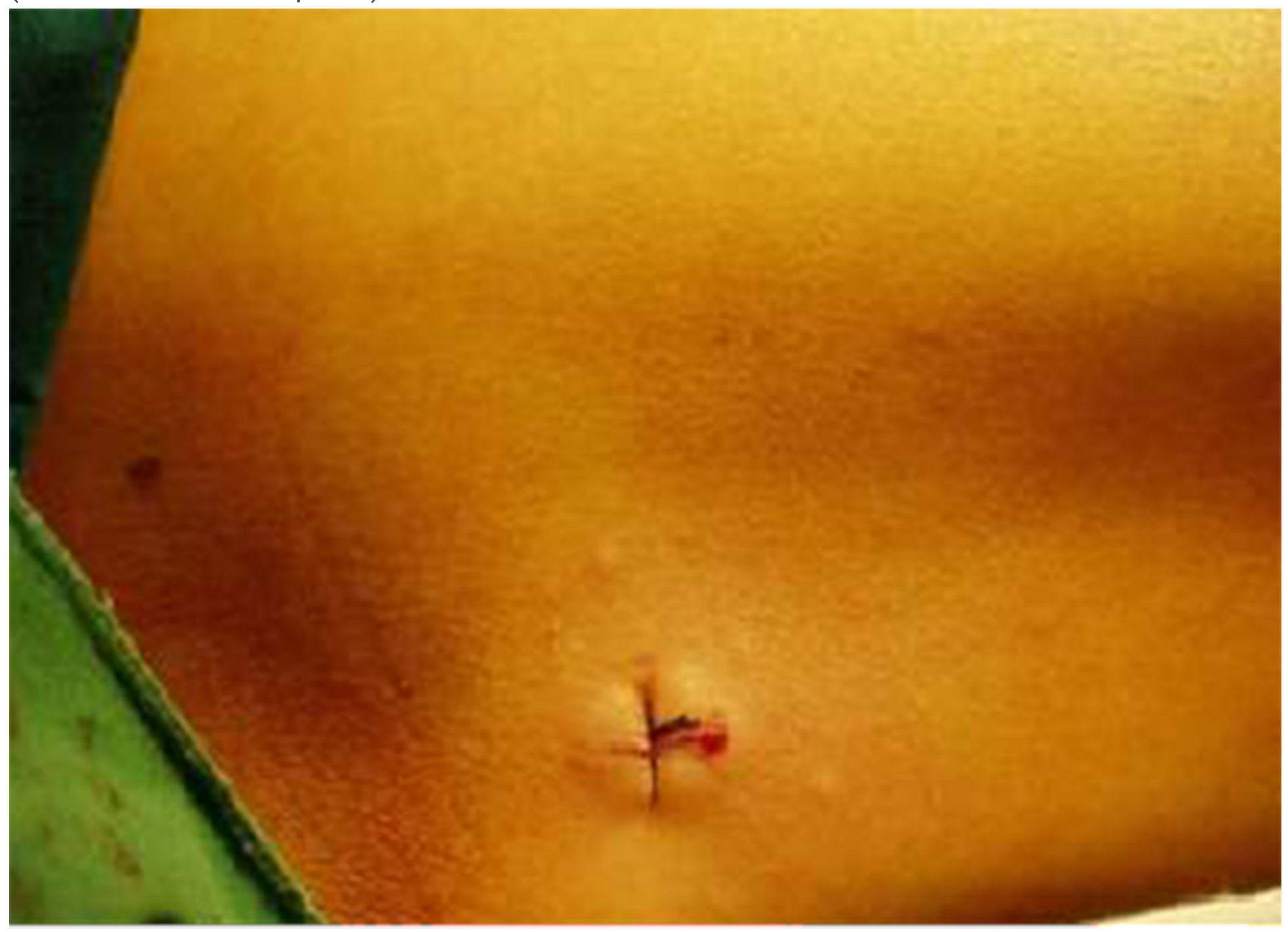

\section{Figure 6}

Sutured minimally invasive incision 